\title{
Le kéfir \\ Caractères physico-chimiques, microbiologiques et nutritionnels. Technologie de production. Une revue
}

\author{
Athéna ZOURARI * et E.M. ANIFANTAKIS \\ Laboratoire de Technologie laitière, Ecole Supérieure d'Agriculture d'Athènes, Grèce
}

\begin{abstract}
Résumé
Le kéfir est un lait fermenté préparé à l'aide d'un levain constitué par les grains de kéfir. Il est produit principalement en URSS et en second lieu dans plusieurs pays, notamment d'Europe, où une législation existe concernant sa qualité et sa fabrication.

Ce produit est caractérisé par la présence d'acide lactique et d'alcool, produits respectivement par des bactéries lactiques et des levures en association avec des bactéries acétiques.

Les grains, composés essentiellement d'un polysaccharide microbien, présentent une structure complexe. Le rôle joué par les micro-organismes dans leur formation et la localisation de ces derniers dans le grain sont discutés.

La production du kéfir à l'échelle industrielle comprend plusieurs étapes. Le traitement du lait et des grains ainsi que les conditions d'incubation, de maturation et de conservation sont d'une grande importance et influencent la qualité du produit. L'optimisation de la production industrielle a amené à remplacer les grains par des levains prêts à l'emploi. D'autres études visent à prolonger le temps de conservation du produit et à appliquer des traitements qui répondront à des demandes diverses.

Le kefir possède les qualités nutritionnelles des autres laits fermentés (digestibilité du lait améliorée par la fermentation, consommation sans problème par les individus intolérant au lactose...), d'où sa popularité et la nécessité d'une étude plus approfondie de ses qualités spécifiques.
\end{abstract}

Mots clés : Kéfir - Laits fermentés - Association - Bactéries lactiques - Levures.

\section{Summary}

Ke ir. Physico-chemical, microbiological and nutritional characters. Production technology. A review.

Kefir is a fermented milk prepared with the kefir grains. It is mainly produced in the USSR and secondly in numerous other countries, mostly in Europe where legislation exists concerning to its quality as well as its production.

\footnotetext{
* Adresse actuelle : INRA, Laboratoire de Microbiologie laitière, 78350 Jouy-en-Josas, France.
} 
This beverage is characterized by the presence of lactic acid and alcohol produced respectively by lactic acid bacteria and yeasts. These two microbial groups predominate associated with acetic acid bacteria.

The grains, which are principally composed of a microbial polysaccharide have a complex structure. They are produced only from grains already existing. The microorganisms involved and their localization in the grain are briefly discussed.

Industrial production of kefir includes several steps. The milk and grains treatment as well as the incubation, ripening and storage conditions play a central role on the quality of the beverage. The attempts to lower the cost of kefir production led to the substitution of grains by ready-to-use starter cultures. Other studies concentrate on the prolongation of the product's shelf-life and the use of new treatments stimulating its consumption.

Kefir possesses the nutritional properties of other fermented milks (improved digestibility compared with milk, consumption without problems by lactose-intolerant individuals...) what justifies both its popularity and the need for a further study of its specific qualities.

Key words : Kefir - Fermented milks - Association - Lactic acid bacteria - Yeasts.

\section{Introduction}

Les laits fermentés ont été développés dans le monde entier, afin de répondre au besoin de prolonger le temps de conservation du lait. Il s'agit de produits traditionnels ; certains ont évolué et de nos jours sont fabriqués à une échelle industrielle au moyen de souches microbiennes sélectionnées et d'équipements modernes. Ce sont des fractions essentielles de l'alimentation de divers peuples d'Europe mais également d'Asie et d'Afrique.

Très tôt, de nombreux chercheurs se sont prononçés très favorablement sur les avantages nutritionnels des produits fermentés qui jouissent d'une grande vogue, leur « image santé » étant nettement positive. Néanmoins, la base scientifique de ces affirmations et, en particulier, de leur influence sur la flore gastro-intestinale et la santé de l'homme, n'est pas fermement établie (Anon., 1983a).

Parmi ces produits, le kéfir, lait fermenté d'origine caucasienne, doit sa particularité et son goût spécifique à une association de bactéries lactiques et de levures. Cette revue présente les différents aspects de la boisson et donne les tendances modernes et les points intéressant la recherche et l'industrie.

\section{Généralités}

Le kéfir est un lait fermenté, acide et alcoolisé, produit principalement à partir des laits de vache, de brebis ou de chèvre à l'aide de "grains de kéfir ».

La région d'origine de cette boisson est le Sud du Caucase où on la prépare jusqu'à nos jours, sous des noms très variés. La dénomination la plus fréquente est « kéfir » qui est d'origine turque (HORVATH, 1968). 
La formation des premiers grains de kéfir et la méthode de préparation utilisée par les anciens peuples du Caucase mêlent les légendes et la réalité, des rapports scientifiques n'existant pas (KEMP, 1984 ; PIDOuX, 1984).

La première publication concernant cette boisson, faite par un médecin russe, G. Dzhogin, en 1867, dans les Travaux de la Société Médicale du Caucase, a été suivie par des dizaines d'autres (Lipatov, 1978). En Europe, la boisson a été introduite vers 1860. L'industrie bavaroise, en 1936, utilisait déjà les grains de kéfir pour la valorisation du lactosérum (Horvath, 1968).

\section{Production et consommation du kéfir}

Les données bibliographiques concernant la production et la consommation internationales du kéfir sont incomplètes. Souvent, on ne le distingue pas d'autres produits laitiers, fait qui rend toute estimation plus difficile (Anon., 1983a).

En Union Soviétique, le kéfir est le plus populaire parmi les laits fermentés et sa vente quotidienne atteint des quantités considérables : on estime à 51 la consommation individuelle et annuelle du kéfir dans ce pays (KosIKOwSKI, 1977). D'après Lipatov (1978), la consommation du kéfir en URSS est passée de 15900 $t$ en 1940 à $1254000 \mathrm{t}$ en 1975. La demande importante est suivie d'une production élevée à l'échelle industrielle. On a rapporté qu'en 1973 une usine à Moscou produisait $100 \mathrm{t}$ de kéfir par jour (LANG et LANG, 1973).

On produit des quantités limitées de kéfir en Tchécoslovaquie, en Pologne, en Hongrie, en Hollande, en RFA, en Suède, en Suisse, en Grèce, aux États-Unis et ailleurs.

En RFA, une enquête a montré qu'en 1976 le kéfir constituait $12 \%$ des boissons de lait fermenté consommées (Anon., 1976). En 1981, ce taux est passé à $18 \%$ (Siegler, 1982).

En Suède, la mise en vente du kéfir date de la moitié de la décennie 1960-1970. En 1985,12 fabricants produisaient à peu près $17000 \mathrm{t}$ de kéfir, qui correspondaient à une consommation individuelle de $1,9 \mathrm{~kg}$ par an (PETTERSSON et al., 1985).

De nos jours, l'augmentation de la production du kéfir devrait répondre à une hausse de la demande pouvant devenir considérable au moyen d'une promotion convenable. La nécessité de la promotion est évidente si l'on considère les facteurs influençant la consommation des laits fermentés dans un pays : la tradition, le climat, le niveau de la technologie utilisée par l'industrie laitière, les différents aspects et le niveau de la consommation du lait et d'autres boissons (LIPATOv, 1978).

\section{Composition chimique}

La composition chimique du kéfir dépend de l'origine et de la composition des grains de kéfir et des laits utilisés. Trois composés présentent des variations : la matière grasse, l'acide lactique et l'alcool. 
Le taux de matière grasse dépend de l'origine du lait utilisé (de vache, de brebis, de chèvre...) et du choix du fabricant (lait entier, écrémé ou partiellement écrémé).

Les taux d'acide lactique rapportés varient entre 0,6 et $0,9 \%$ (JACQUET et THEVENOT, 1961) par suite des différentes méthodes de préparation et de la microflore utilisées.

On note des variations considérables du taux d'alcool, entre 0,01 \% (KoroLEVA, 1982) et $1 \%$ (KosiKowsKi, 1977). Glaeser (1981) a trouvé que le kéfir industriel en RFA renfermait moins de $0,01 \%$ d'alcool, le kéfir industriel produit dans l'Europe de l'Est 0,01 à $0,04 \%$ et le produit traditionnel 0,03 à $1 \%$. De plus, des échantillons de kéfir industriel allemand ont montré un contenu en alcool de 0,002-0,005\%, bien inférieur à ce que l'on rapporte dans la littérature (Glaeser et Beiter, 1979). On peut donc supposer que, dans divers pays, la variabilité de la composition du kéfir reflète des différences éventuelles du processus de fabrication.

D'après KosiKowski (1977), une proportion optimale de 3:1 entre le diacétyle et l'acétaldéhyde définit l'arôme typique du kéfir : l'alcool et l'acétone y ont une contribution limitée. On considère que le propionaldéhyde, le butanone-2, le npropanol, l'alcool isoamylique et l'acide acétique sont des substances également importantes, mais leur taux, comme celui de l'alcool varient considérablement durant la maturation (Görner et al., 1970 ; Rosi et Rossi, 1978).

Le kéfir est le seul lait fermenté subissant une double fermentation lactique et alcoolique pour lequel une législation existe dans plusieurs pays, comme l'URSS, la Pologne, la RFA, Israël, le Brésil, l'Autriche, la Norvège (Desez, 1964 ; Anon. b). Ces législations donnent des standards de composition, de qualité, de conditions de préparation et d'emballage devant être respectés par les fabricants.

\section{Aspects microbiologiques du kéfir}

\section{A. Les grains de kéfir}

Les grains de kéfir sont des masses gélatineuses, irrégulières, de taille variable, insolubles dans l'eau et dans la plupart des solvants. A l'état frais, ils sont blanchâtres et rappellent le " pop-corn » ou les inflorescences du chou-fleur. A l'état $\mathrm{sec}$, ce sont des masses dures, jaunes, au sein desquelles se trouvent les microorganismes du kéfir dont la vitalité dépend des conditions de dessèchement et de conservation des grains. Dans le lait, les grains gonflent et blanchissent.

La composition chimique globale de grains de kéfir d'origines diverses est donnée dans le tableau 1 (Ottogalli et al., 1973). LA Riviere et al. (1967) ont constaté que presque $50 \%$ du polysaccharide représentant $24 \%$ du poids sec de grains de kéfir d'origine hollandaise était précipité par addition d'alcool. Son hydrolyse produisait seulement du D-glucose et du D-galactose dans une proportion de 1:1. Ce polysaccharide n'a été trouvé que dans les grains de kéfir et pour cette raison, ils l'ont appelé kéfirane. 


\section{TABLEAU 1}

Composition chimique des grains de kéfir d'origines diverses (1) (OtTOGALLl et al., 1973) Chemical composition of kefir grains from different origin ${ }^{(1)}$ (OtTogallı et al., 1973)

\begin{tabular}{|c|c|c|c|c|c|c|}
\hline \multirow{2}{*}{$\begin{array}{c}\text { Pays } \\
\text { d'origine }\end{array}$} & \multirow[b]{2}{*}{ Eau } & \multirow{2}{*}{$\begin{array}{l}\text { Mat. } \\
\text { sèche }\end{array}$} & \multicolumn{4}{|c|}{ Composition de la matière sèche } \\
\hline & & & $\begin{array}{l}\text { Mat. } \\
\text { grasse }\end{array}$ & Prot. ${ }^{(2)}$ & SENA $^{(3)}$ & Cendres \\
\hline URSS & 89,5 & 10,5 & 2,8 & 30,3 & $59,3(40,1)$ & 7,6 \\
\hline Yougoslavie & 88,9 & 11,1 & 4,3 & 31,4 & $57,2(42,1)$ & 7,2 \\
\hline Yougoslavie & 90,0 & 10,0 & 3,5 & 34,5 & $55,0(40,1)$ & 7,0 \\
\hline Bulgarie & 90,6 & 9,4 & 3,5 & 34,4 & $53,4(35,8)$ & 8,7 \\
\hline
\end{tabular}

(1) Tous les résultats sont exprimés en $\%$.

(1) All the results are expressed in percent.

(2) Protéines.

(2) Proteins.

(3) Substances extractibles non azotées. Entre parenthèses figurent les estimations des sucres réducteurs, après hydrolyse sous conditions acides.

(3) Nitrogen free extract. Between brackets the reducing sugars after acid hydrolysis.

\section{B. La microflore du kéfir et son évolution durant la fermentation}

Dans les grains de kéfir on est en présence d'une association entre plusieurs groupes microbiens : des streptocoques mésophiles, des leuconostocs, des lactobacilles mésophiles ou thermophiles, des levures et des bactéries acétiques (tabl. 2).

Des variations de la microflore résultent de l'origine différente des grains étudiés et sont plus accentuées dans le cas des levures. Ainsi, hormis les levures isolées des grains de kéfir citées dans le tableau 2, on en a isolé les espèces : Candida kefir (Moulin et al., 1977), Kluyveromyces lactis, Candida valida, Brettanomyces anomalus, Saccharomyces unisporus (OHARA et al., 1977), Torulopsis holmii (IWASAwa et al., 1982), Torulopsis kefir, Saccharomyces florentinus et Saccharomyces carlsbergensis (MERILAINEN, 1984).

KOSIKOWSKI (1977) rapporte que les levures représentent 5 à $10 \%$ de la population microbienne totale et que la présence de Geotrichum candidum, qui couvre souvent la surface des grains ne paraît pas nuire à la qualité du produit.

Il faut noter que selon le document $\mathbf{4 7}$ de la Fédération Internationale de Laiterie (Anon., 1969), les ferments acceptés pour la préparation du kéfir sont les levures fermentant le lactose, les lactobacilles, les streptocoques lactiques et les grains de kéfir.

Chaque groupe microbien, suivant ses propriétés physiologiques, prédomine pendant une phase différente de la préparation du kéfir. Les streptocoques lactiques mésophiles produisent de l'acide lactique au début de la fermentation. Ensuite, le développement des lactobacilles augmente l'acidité qui diminue la population des streptocoques. Les leuconostocs se multiplient plus lentement. Les 
TABleau 2

La microflore du kéfir selon divers auteurs

The kefir microflora according to different authors

\begin{tabular}{|c|c|c|c|c|}
\hline & Référence & Bactéries lactiques & Levures & Autres groupes \\
\hline $\begin{array}{l}\text { Ros } \\
\text { Ros } \\
(197\end{array}$ & $\begin{array}{l}(1978 a, b) \\
\text { t Rossi }\end{array}$ & $\begin{array}{l}\text { Str. durans } \\
\text { Lb. brevis } \\
\text { Leuc. } \\
\text { mesenteroides }\end{array}$ & $\begin{array}{l}\text { Sacch. cerevisiae } \\
\text { Sacch. delbrueckii }\end{array}$ & $\begin{array}{l}\text { Acetobacter } \\
\text { aceti }^{(5)}\end{array}$ \\
\hline$\widehat{m}$ & URSS & $\begin{array}{l}\text { Str. lactis } \\
\text { Leuc. kefir } \\
\text { Lb. brevis } \\
\text { Lb. acidophilus }\end{array}$ & Sacch. lactis & 一 \\
\hline 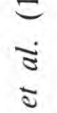 & Yougoslavie & $\begin{array}{l}\text { Str. lactis } \\
\text { Lb. brevis } \\
\text { Lb. acidophilus }\end{array}$ & Candida tenuis & $\begin{array}{l}\text { Acetobacter } \\
\text { rancens }\end{array}$ \\
\hline \begin{tabular}{l}
$\bar{Z}$ \\
\multirow{6}{0}{} \\
0
\end{tabular} & Yougoslavie & $\begin{array}{l}\text { Str. lactis } \\
\text { Lb. brevis } \\
\text { Lb. acidophilus } \\
\text { Leuc. kefir }\end{array}$ & $\begin{array}{l}\text { Sacch. lactis } \\
\text { Sacch. carlsbergensis }\end{array}$ & - \\
\hline 0 & Bulgarie & $\begin{array}{l}\text { Str. lactis } \\
\text { Lb. brevis } \\
\text { Lb. acidophilus }\end{array}$ & $\begin{array}{l}\text { Candida } \\
\text { pseudotropicalis }\end{array}$ & $\begin{array}{l}\text { Bacillus } \\
\text { subtilis }\end{array}$ \\
\hline $\begin{array}{l}\text { Kos } \\
(197\end{array}$ & DWSKI & $\begin{array}{l}\text { Lb. caucasicus } \\
\text { Leuconostoc spp. } \\
\text { streptocoques } \\
\text { lactiques }\end{array}$ & $\begin{array}{l}\text { Sacch. kefir } \\
\text { Torula kefir }\end{array}$ & $\begin{array}{l}\text { Geotrichum } \\
\text { candidum }\end{array}$ \\
\hline
\end{tabular}

(1) $10^{6} / \mathrm{ml}$ de boisson.

(1) $10^{6} / \mathrm{ml}$ of beverage.

(2) $10 \% / \mathrm{ml}$ de boisson et $\mathrm{g}$ de grains.

(2) $10^{6} / \mathrm{ml}$ of beverage and $\mathrm{g}$ of grains.

(3) $10^{5} / \mathrm{ml}$ de boisson et $\mathrm{g}$ de grains.

(3) $10^{5} / \mathrm{ml}$ of beverage and $\mathrm{g}$ of grains.

(4) Levures: $10^{5} / \mathrm{ml}$ de boisson et $10^{6} / \mathrm{g}$ de grains $(34 \%$ Sacch. cerevisiae et $66 \%$ Sacch. delbrueckii).

(4) Yeasts: $10^{5} / \mathrm{ml}$ of beverage $10^{6} / \mathrm{g}$ of grains $34 \%$ Sacch. cerevisiae and $66 \%$ Sacch. delbrueckii).

(5) $10^{3} / \mathrm{ml}$ de boisson et $10^{2} / \mathrm{g}$ de grains.

(5) $10^{3} / \mathrm{ml}$ of beverage and $10^{2} / \mathrm{g}$ of grains.

(6) Nom rejeté (RogosA, 1974).

(6) Rejected name (RoGOSA, 1974).

- Ce groupe microbien n’a pas été détecté.

- This microbial group was not detected.

levures et les bactéries acétiques se développent plus tardivement par rapport aux bactéries lactiques (Koroleva, 1982).

Par conséquent, les changements de la composition du kéfir sont plus prononcés au début de la fermentation. Il s'agit d'une augmentation considérable de l'acidité volatile, des substances azotées simples et du $\mathrm{CO}_{2}$; le taux d'alcool reste 
faible. La quantité d'acide orotique diminue puisque les microorganismes en consomment pour la synthèse des nucléotides. Il en est de même pour l'acide citrique, probablement à cause de la production de diacétyle et d'acétoïne qui en dérivent (GAWel et GromadKa, 1978).

\section{Relations entre les microorganismes du kéfir}

Le kéfir représente une association typique de groupes microbiens ayant des demandes différentes pour les facteurs de croissance. Plusieurs relations particulières de dépendance ont été mises en évidence.

Les lactobacilles et les levures sont étroitement liés à bénéfices réciproques. Les levures produisent probablement des facteurs de croissance tels que la riboflavine et l'acide folique qui paraissent indispensables pour la synthèse d'un polysaccharide bactérien participant à la structure du grain. Ainsi, elles interviennent dans le processus de la formation d'un nouveau grain. A titre d'exemple, Lactobacillus brevis, qui produit du polysaccharide en présence d'extrait de levure, ne se développe dans du lait non supplémenté qu'en présence de Saccharomyces delbrueckii, levure isolée du kéfir (LA Riviere et al., 1967). L'importance des levures est aussi confirmée par le fait que le poids des grains de kéfir cultivés dans du lactosérum renforcé avec de l'extrait de levure a présenté une augmentation bien supérieure à celle obtenue dans le lait ou le lactosérum sans supplément (HiRota, 1987).

Les levures, en s'autolysant, libèrent des acides aminés et des facteurs de croissance permettant la survie et la multiplication des lactobacilles qui ont des difficultés à utiliser les acides aminés et la caséine du lait. La synthèse par les bactéries lactiques d'une B-galactosidase (lactase) qui hydrolyse le lactose favorise les levures, si celles-ci ne peuvent pas utiliser ce sucre contrairement au glucose et/ou au galactose qui en résultent (JACQUET et THEVENOT, 1961 ; Rosi, 1978a ; Rosi et Rossi, 1978).

Le rôle des bactéries acétiques paraît aussi intéressant (Rosı et Rossı, 1978). Elles synthétisent la vitamine $B_{12}$, à la différence des bactéries lactiques et des levures. Les bactéries acétiques isolées du kéfir oxydent l'acide lactique produit durant la fermentation lactique et utilisent l'éthanol produit par les levures et les bactéries lactiques hétérofermentaires, pour la biosynthèse de l'acide acétique.

Le tamisage du caillé sélectionne la population des grains, puisque les microorganismes qui n'y sont pas bien attachés restent dans le lait fermenté (LA RIVIERE et al., 1967).

Koroleva (1982) rapporte que les relations établies entre les microorganismes du kéfir sont responsables de certaines propriétés d'intérêt industriel. Ces microorganismes ne répondent pas aux variations saisonnières de la qualité du lait (KoroLEVA et al., 1978a), ni à la présence d'antibiotiques et d'autres inhibiteurs (présents d'habitude en quantités détectables) et ils résistent aux bactériophages. Cependant, les deux derniers caractères ne sont pas cités par d'autres auteurs.

\section{Structure et formation des grains de kéfir}

L'étude des grains de kéfir à l'aide du microscope électronique (BotTazzı et BıANCHI, 1980) a révélé que la partie périphérique du grain était peuplée presque 


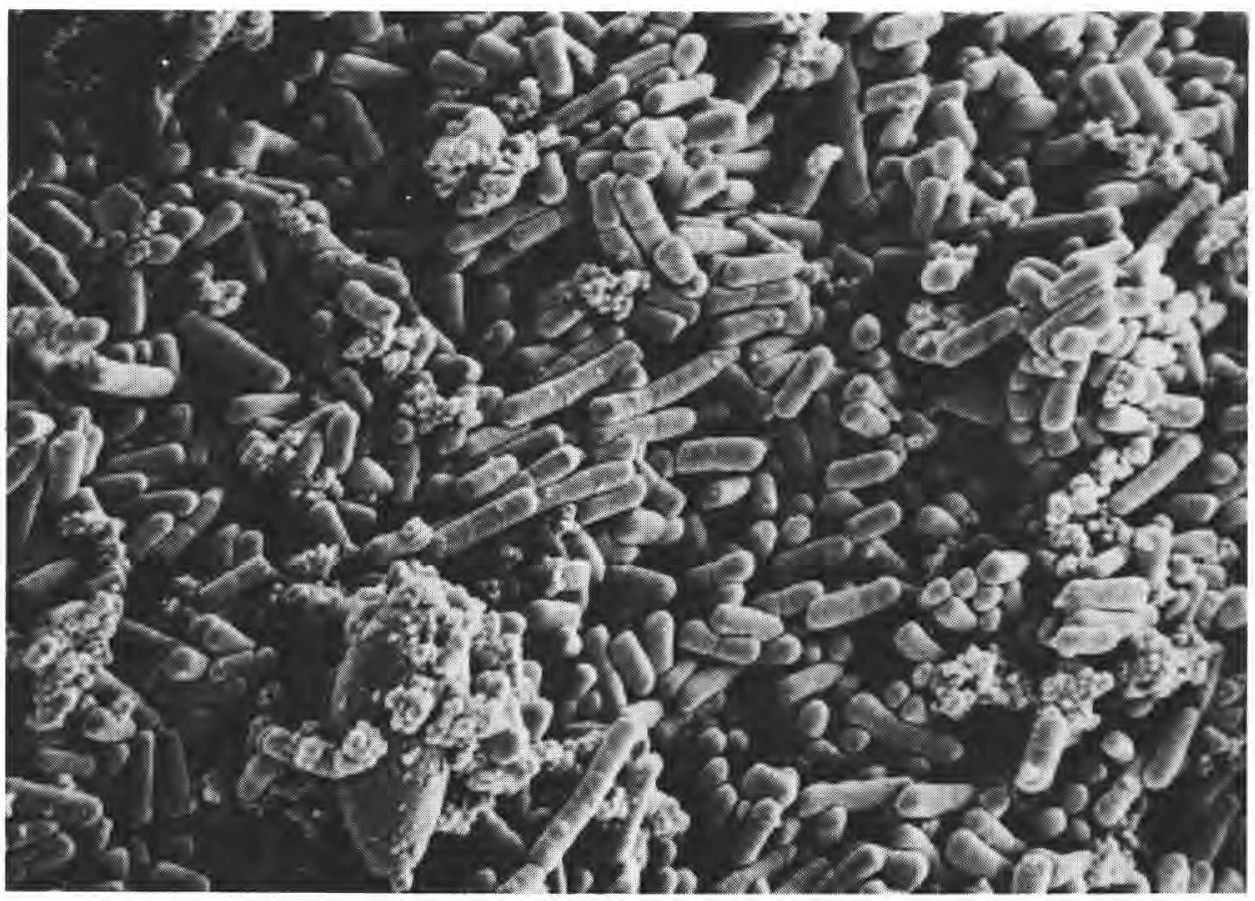

Fig. 1

Prédominance des bactéries à la partie périphérique du grain de kéfir (x3040; BotTazzi et BIANCHI, 1980).

Predominance of rod-shaped bacteria in the peripheral layer of the kefir granule $(x 3040$; BOTTAZZI and BIANCHI, 1980).

\section{Fig. 2}

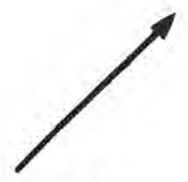

Bactéries et levures à la partie intermédiaire du grain de kéfir situées sur la matrice lamellaire qui forme une masse spongieuse (x3040; BotTAZZI et BIANCHI, 1980).

Rod-shaped bacteria and yeasts in the median part of the kefir granule on the lamellar matrix which forms a spongy mass (x3040; BOTTAZZI and BIANCHI, 1980).

Fig. 3

Prédominance des levures au centre du grain de kéfir; elles sont placées sur une matrice lamellaire fortifiée par des cordons (x3040; BotTaZzI et BIANCHI, 1980).

Predominance of yeasts at the centre of the kefir granule on the lamellar matrix strengthened by cordons (x3040; BotTAZZI and BIANCHI, 1980). 

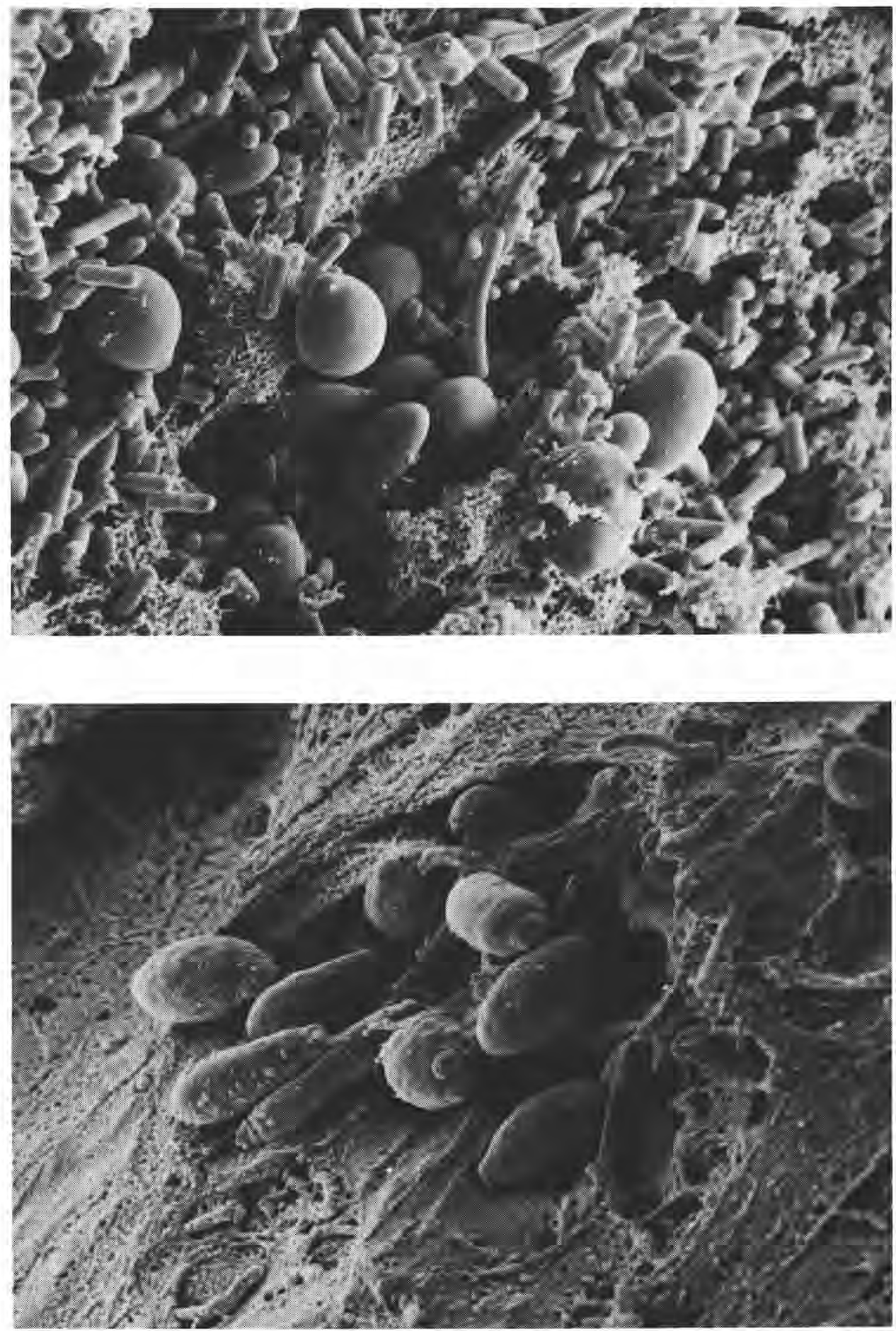
exclusivement de bactéries, les levures étant rares (fig. 1). Vers l'intérieur du grain, la composition de la microflore évolue progressivement en faveur des levures qui prédominent au centre (fig. 2 et 3 ). Les microorganismes se situent dans la matière spongieuse du grain qui semble les soutenir et qui se présente sous forme d'une matrice lamellaire fortifiée par des cordons plus denses. La population de la partie centrale est plus faible que celle de la partie périphérique du grain.

Les levures (en particulier) et les lactobacilles forment des accumulations (《 microcolonies »), à la différence des streptocoques. A l'intérieur du grain, les cellules des lactobacilles et des levures ne sont pas associées mais enrobées dans une matière gluante de polysaccharide microbien (MoLSKA et al., 1980).

La matrice insoluble du grain est composée d'un polysaccharide ramifié, probablement produit par les lactobacilles du centre du grain. La population microbienne du centre diffère morphologiquement de celle de la surface et pose des difficultés pour l'isolement de souches. Ces organismes sont étroitement liés avec le polysaccharide par leurs parois cellulaires. L'insolubilité du polysaccharide limiterait la diffusion des substances nutritives et des métabolites microbiens. Ainsi, les microorganismes inclus meurent, devenant des parties inséparables de la structure du grain (Marshall et Cole, 1984).

Les grains de kéfir représentent, à l'heure actuelle encore, un défi à surmonter pour les microbiologistes qui les étudient, puisque tous les efforts pour les faire se former à partir de souches isolées du produit ont jusqu'à présent été voués à l'échec. Les grains sont formés seulement à partir de grains déjà existants.

La culture des bactéries isolées du kéfir dans le lait entraîne son acidification mais ne conduit pas à la formation des grains. Probablement, un « support " de la microflore, représenté par le grain lui-même, est-il nécessaire. Peut-être, les levures en fonction de leur localisation dans la partie centrale et les canaux périphériques du grain constituent-elles les points de prolifération qui sont à l'origine d'un noyau secondaire. Celui-ci, détaché, donnera naissance à un nouveau grain . Le mécanisme de la formation des grains dépendrait aussi du lait utilisé et en particulier du traitement thermique subi (Rosi et Rossi, 1978).

La biosynthèse du polysaccharide qui confère au grain son élasticité est attribuée à Lactobacillus brevis (LA RIVIERE et al., 1967). Cette propriété est rapidement perdue pendant l'isolement des souches et c'est pour cela qu'elle n'a pas été détectée par d'autres chercheurs. Cependant, OtTogalli et al. (1973) attribuent la formation du kéfirane à Lactobacillus acidophilus.

Rosı et Rossi (1981) ont obtenu la biosynthèse du kéfirane dans le lait par une souche de Streptobacterium, en présence d'autolysat de levure et de cellules vivantes de Saccharomyces delbrueckii. Selon les mêmes chercheurs, la synthèse du kéfirane dans un milieu synthétique nécessite la présence du lactose (à un taux de 3 à $9 \%$ ) ou du saccharose, un $\mathrm{pH}$ voisin de 6 et une température de $30^{\circ} \mathrm{C}$ environ.

\section{La préparation du kéfir}

La fabrication du kéfir est assez particulière et difficile à cause de la complexité de la composition microbiologique du levain qui résulte de l'utilisation 

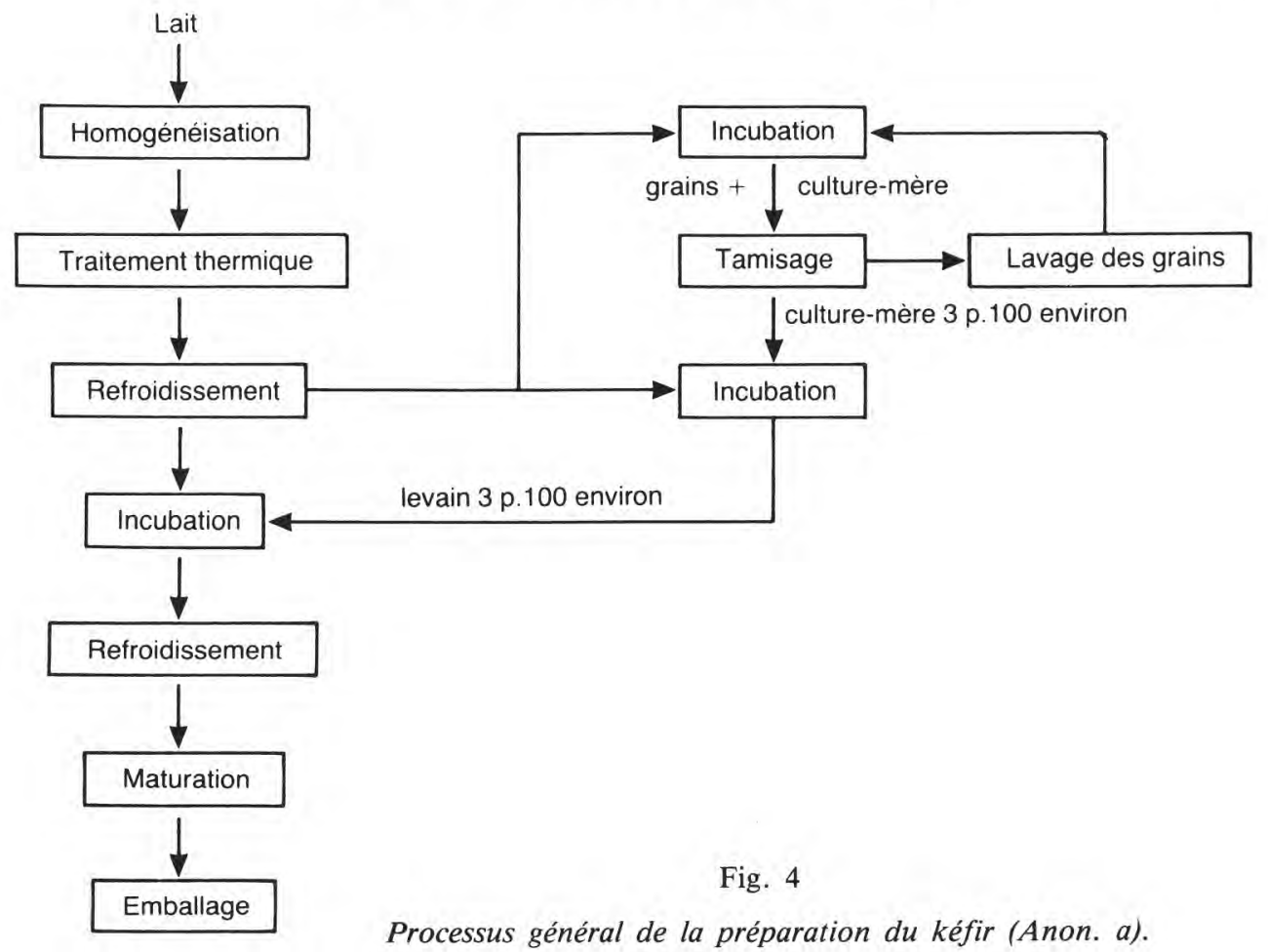

levain 3 p.100 environ

Fig. 4

Processus général de la préparation du kéfir (Anon. a).

General process of kefir production (Anon. a).

des grains de kéfir. La stabilité des conditions de culture des grains, le type, la qualité et le traitement du lait, la quantité du levain, la température et la durée de fermentation, l'équipement mécanique pour la manipulation du caillé sont des points critiques de la fabrication qu'on doit surveiller continuellement (LIPATOV, 1978).

Le procédé général de la préparation du kéfir (fig. 4, Anon. a) comprend les étapes suivantes :

(a) Le lait. On utilise essentiellement le lait de vache, de brebis ou de chèvre.

(b) La préparation du levain. On le prépare avec du lait entier ou du lait écrémé, homogénéisé et pasteurisé à une température élevée. On peut distinguer deux étapes successives :

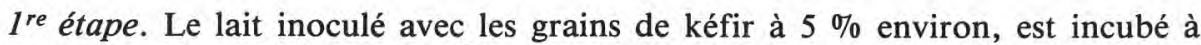
une température avoisinant $23{ }^{\circ} \mathrm{C}$ pendant $20 \mathrm{~h}$ à peu près, brassé de temps en temps suivant la tradition du Caucase, pour améliorer l'homogénéité et l'onctuosité du produit. Après l'obtention du $\mathrm{pH}$ convenable, les grains récupérés par tamisage et lavés avec de l'eau bouillie et refroidie sont inoculés de nouveau dans du lait. 
$2^{e}$ étape. Du lait pasteurisé et homogénéisé, inoculé avec la culture tamisée (" culture-mère »), est ensuite incubé dans les conditions mentionnées. On obtient ainsi le levain, utilisé à un taux de 3 à $5 \%$ pour la préparation du kéfir.

Les conditions de conservation des grains sont très importantes. A l'état frais, dans de l'eau ou du lactosérum stériles, les grains gardés au froid $\left(4{ }^{\circ} \mathrm{C}\right)$ perdent leur activité en 8 à 10 jours. Séchés à température ambiante pendant 36 à $48 \mathrm{~h}$ et conservés au froid, ils retrouvent leur activité totale après 2 à 3 repiquages dans le lait, même après 12 à 18 mois de conservation (KOSIKowsK1, 1977). Récemment, la lyophilisation et la congélation sont des techniques qui ont été également proposées pour la conservation des grains.

(c) Homogénéisation du lait. Une pression élevée et le préchauffage du lait à la température d'homogénéisation (par exemple $15 \mathrm{MPa}$ et $70^{\circ} \mathrm{C}$ ) assurent la bonne texture du produit.

(d) Traitement thermique du lait. Il est d'une importance considérable pour la qualité du produit. Il vise à la dénaturation complète des albumines et des globulines du lait, nécessaire pour que la viscosité et la texture soient convenables. Réalisé à une température élevée pour une période prolongée, le traitement thermique permet une rétention aqueuse importante qui réduit la synérèse et augmente la viscosité. Parallèlement, il tue la plupart des microorganismes et facilite l'utilisation des protéines du lait par la microflore.

On cite dans la bibliographie deux sortes de traitement thermique : à $90-95{ }^{\circ} \mathrm{C}$ pendant un temps qui varie de 2 à $30 \mathrm{mn}$ et à $85-87^{\circ} \mathrm{C}$ pour $5 \mathrm{mn}$ à $1 \mathrm{~h}$.

(e) Refroidissement du lait - Incubation. On propose un refroidissement à $20-25{ }^{\circ} \mathrm{C}$, une addition de 2 à $8 \%$ de levain (couramment $5 \%$ ) et une incubation à $20-22{ }^{\circ} \mathrm{C}$ pendant 8 à $20 \mathrm{~h}$.

(f) Refroidissement du caillé. Lorsque le $\mathrm{pH}$ est situé entre 4,5 à 4,6, le produit est refroidi à $4-6{ }^{\circ} \mathrm{C}$ en $20 \mathrm{mn}$ (au maximum) pour empêcher un abaissement supplémentaire du $\mathrm{pH}$. Il faut éviter l'incorporation d'air dans le caillé, qui augmenterait le risque de stratification du produit.

(g) Maturation et emballage. Après refroidissement, le produit est transporté dans un tank pour sa stabilisation, pendant laquelle les protéines dénaturées absorbent une quantité maximale d'eau. Ensuite, le produit est pompé doucement en évitant tout dommage au caillé, et placé dans une chambre froide pour sa maturation. Des températures de maturation de $8{ }^{\circ} \mathrm{C}$ à $16^{\circ} \mathrm{C}$, pendant $12 \mathrm{~h}$ à 6 jours sont couramment pratiquées. A la sortie du froid, le $\mathrm{pH}$ doit être égal à $4,3-4,4$.

Ce qui différencie le kéfir des autres laits fermentés, c'est la production de $\mathrm{CO}_{2}$ durant la fermentation alcoolique (Siegenthaler et RitTer, 1964). La production du gaz se poursuit, en général, pendant la conservation à des températures basses et peut exercer une forte pression sur l'emballage. On doit donc utiliser des récipients et des bouchons résistant à une pression élevée, comme les bouchons à ressort qui permettent l'évacuation d'une certaine quantité de $\mathrm{CO}_{2}$. Des pots en plastique fermés avec des couvercles thermo-soudés et munis de quelques petits trous, offrent le même avantage et sont employés industriellement, en Suisse par exemple. 
Aujourd'hui, le kéfir est vendu dans des bouteilles en verre à bouchons métalliques, dans des emballages en carton ou dans des pots en plastique; le problème de la résistance est diminué par une fermentation alcoolique limitée et par les couvercles décrits ci-dessus.

\section{Facteurs influençant la qualité du kéfir}

La qualité du kéfir devrait prendre en compte l'ensemble de ses propriétés, à savoir la composition chimique, la microflore (du point de vue quantitatif et qualitatif), les qualités rhéologiques et les caractères organoleptiques. Ainsi, est-elle liée à l'origine, à la composition et à la qualité du lait et des grains, mais aussi aux conditions de production et à la technologie utilisée.

La composition et les caractéristiques biochimiques du kéfir dépendent de la composition de la microflore, puisqu'elles résultent de son activité métabolique. Par conséquent, elles sont influencées par le temps d'incubation en présence des grains, la proportion entre les grains et le lait (dont la diminution favorise la plupart des microorganismes), l'utilisation simultanée des grains et du levain déjà prêt et le lavage des grains. Ce dernier, en éliminant une quantité importante des microorganismes acidifiants, ralentit l'acidification et réduit les taux d'acide lactique et d'acides volatils (Koroleva et Bavina, 1970).

L'utilisation des grains augmente la population de tous les groupes microbiens dans le produit à l'exception des lactobacilles thermophiles qui sont favorisés par l'utilisation du caillé (Koroleva et al., 1978a).

Le temps et les conditions de conservation déterminent également la composition de la microflore du produit. Pendant la conservation à basse température (à 2-4 ${ }^{\circ} \mathrm{C}$ comme à $6-8{ }^{\circ} \mathrm{C}$ ) l'évolution de la microflore est lente. Après 7 jours de conservation, sa composition n'est pas très diversifiée. Par contre, une conservation à $18-20^{\circ} \mathrm{C}$ entraîne des modifications prononcées (Koroleva et al., 1978b).

Les qualités organoleptiques du kéfir sont associées aux modifications chimiques résultant de l'activité microbienne des grains et du levain ; elles dépendent donc du processus de fermentation et de maturation.

Comme la qualité du kéfir est liée aux taux d'acide lactique et d'alcool produits, les fermentations correspondantes doivent être en équilibre. Ceci est assuré par le choix d'une température d'incubation convenable. La fermentation lactique est favorisée à température élevée $\left(26^{\circ} \mathrm{C}-35^{\circ} \mathrm{C}\right)$, à la différence de la fermentation alcoolique qui est favorisée à basse température $\left(5^{\circ} \mathrm{C}-15^{\circ} \mathrm{C}\right)$. Par conséquent, les températures comprises entre $18{ }^{\circ} \mathrm{C}$ et $20^{\circ} \mathrm{C}$ sont les meilleures pour l'équilibre recherché (Horvath, 1968 ; Petricic et al., 1977).

Le traitement du lait (chauffage, homogénéisation) joue un rôle important dans la texture et les qualités rhéologiques du produit. La texture dépend considérablement du traitement thermique du lait (BERZHINSKAs et al., 1978). Une température élevée conduit à un caillé plus stable. Par exemple, la pasteurisation à $87^{\circ} \mathrm{C}$ suivie d'une réduction de la température à $77^{\circ} \mathrm{C}$ maintenue pendant $30 \mathrm{mn}$ puis d'un réchauffement à $87^{\circ} \mathrm{C}$ (« multiple stage pasteurization ») favorise la 
dénaturation des protéines du sérum. Elle augmente la dispersion des micelles de la caséine améliorant ainsi la stabilité et la viscosité du caillé tout en réduisant la synérèse. L'homogénéisation du lait à $3,5 \%$ de matière grasse a donné un produit ayant une acidité augmentée de 10 à $20 \%$, un temps de conservation supérieur de 50 à $60 \%$ et des qualités organoleptiques améliorées (SzAKALY et al., 1982). Ceci est dû à une meilleure texture du caillé et à une synérèse réduite ; parallèlement, la montée de la crème est évitée.

L'augmentation du taux de matière grasse de $0,06 \%$ à $2 \%$ améliore les qualités organoleptiques et la texture du produit (PIECHOCKA et al., 1977).

Dans le cas du kéfir brassé, la viscosité dépend considérablement du $\mathrm{pH}$ et de la méthode de refroidissement pendant la maturation. Le meilleur produit requiert un brassage à $\mathrm{pH} 4,5-4,4$ et un refroidissement progressif sans brassage (KoRovKINA et al., 1976).

\section{Innovations et nouvelles tendances dans la production industrielle du kéfir}

Les Soviétiques possèdent, sans nul doute, la meilleure expérience de la production industrielle du kéfir qui doit résoudre deux problèmes principaux : le danger de contaminations et le coût élevé résultant de l'utilisation des grains, ainsi qu'un temps limité de conservation du produit.

Par conséquent, on a surtout essayé de remplacer les grains par un levain conduisant à un produit de qualité supérieure. Divers chercheurs ont obtenu des résultats satisfaisants avec des levains contenant des souches pures isolées du kéfir, dans différentes proportions (VAYSSIER, 1978 ; KramkowsKa et al., 1982 ; DuitsCHAEVER et al., 1987).

Les levains lyophilisés à usage industriel présentent des avantages évidents par rapport au levain traditionnel. Pouvant être conservés 6 mois au moins à une température de $-18{ }^{\circ} \mathrm{C}$, ils assurent :

- un traitement plus facile du levain (risque de contaminations et de modifications réduit, en particulier durant la conservation);

- une stabilité de l'activité du levain, de la qualité du produit et du nombre des levures ;

- la possibilité de modifier la composition du levain pour satisfaire des demandes différentes (Pettersson, 1984 ; Pettersson et al., 1985).

Des cultures prêtes à l'emploi sont déjà offertes aux industries par des laboratoires spécialisés.

La lyophilisation peut prolonger le temps de conservation du produit. Du kéfir concentré et ensuite lyophilisé aurait après sa reconstitution, toutes les qualités microbiologiques et biochimiques du produit de référence (AleKSEEV et Gouliaev, 1981 ; Gulgo et al., 1981). En ce qui concerne les conditions optimales de lyophilisation du kéfir, on a proposé une vitesse de réfrigération de $1,5 \cdot 10^{-2}{ }^{\circ} \mathrm{C} / \mathrm{s}$, une température finale de congélation de $-15^{\circ} \mathrm{C}$ à $-18^{\circ} \mathrm{C}$, une température 
maximale en fin de déshydratation de $35^{\circ} \mathrm{C}$ à $38^{\circ} \mathrm{C}$ et une humidité finale de 3 à $4 \%$ (Gouliaev et Alexeiev, 1982).

Des traitements divers ont été proposés afin de répondre aux demandes différentes :

- L'addition de morceaux, du jus, de la pulpe ou des sirops de fruits, des arômes ou du sucre paraît indispensable pour que les Anglo-Saxons acceptent mieux le kéfir (Kemp, 1984 ; Marshall et Cole, 1985).

- Enrichis en vitamines, ayant des qualités nutritionnelles améliorées ou sous forme de mélanges spéciaux, des produits à base de kéfir peuvent être consommés par les enfants et ont déjà donné des résultats satisfaisants en URSS (IvanOva et al., 1980 ; KlYAvinYa, 1980). L'enrichissement en vitamines est surtout orienté vers les vitamines dont le taux dans le kéfir est faible, comme la vitamine $\mathrm{B}_{12}$. Des souches diverses de Propionibacterium ( $P$. shermanii, $P$. petersonii, $P$. pituitosum) ont été introduites dans la microflore du kéfir ou utilisées parallèlement aux grains. De cette façon, le contenu en vitamine $\mathrm{B}_{12}$ du produit a considérablement augmenté (jusqu'à 50 fois à peu près) sans modifications de ses caractères organoleptiques (Karlin, 1965 ; Roczniakova et al., 1974 ; Cerna et Hrabova, 1977).

- L'addition de sels de calcium ou de préparations protéiques (caséinate de sodium, protéines du sérum, lactosérum ultrafiltré ou déshydraté) augmente le contenu protéique du produit ainsi que sa valeur nutritive et améliore sa texture et son homogénéité (Rosi et Rossi, 1980 ; CHOJNOWSKI et al., 1978, 1981 ; BONDAREV, 1981 ; Bogdanova et al., 1982). De cette façon, on peut éviter l'utilisation de divers stabilisants (Eller, 1983).

- Une perspective probable est l'utilisation de lait délactosé par fermentation avec une levure ou par l'addition de préparations enzymatiques (lactases) (Rossi et al., 1978).

- La fabrication du kéfir à partir de farines végétales (soja, petit-pois, haricot, lupin) mélangées ou non avec du lait et des concentrés de protéines du sérum a été également étudiée (Rossi et ClemenTI, 1983). Des produits de ce type, riches en protéines et ayant des caractères organoleptiques améliorés, conviendraient aux pays du Tiers Monde dont l'alimentation est basée sur les produits végétaux.

\section{Les qualités nutritionnelles du kéfir}

Par suite de son utilisation par les microorganismes, le lactose contenu dans le kéfir diminue de $30 \%$ (ALm, 1982a). Une B-galactosidase contenue dans le kéfir a été purifiée et caractérisée par des chercheurs japonais (HIROTA et KIKUCHI, 1976). Sa présence pourrait expliquer le fait que le kéfir est consommé sans problème par les personnes intolérantes au lactose. Cependant, le rôle effectif des ß-galactosidases bactériennes dans la digestion du lactose au niveau de l'intestin n’a pas été rigoureusement démontré (Anon., 1983b). 
L'acide lactique est presque exclusivement sous la forme de son isomère $\mathrm{L}(+)$ (Wiesner et al., 1975 ; GLAESER, 1981 ; Alm, 1982d), à la différence du yoghourt qui contient aussi l'isomère $\mathrm{D}(-)$ ( 40 à $50 \%$ de l'acide lactique total). Sur ce point, il faut tenir compte que l'isomère $\mathrm{D}(-)$ absorbé en trop grande quantité peut provoquer des troubles du métabolisme. Cependant, cela ne se produit que dans le cas d'un régime extrêmement déséquilibré (Anon., 1983b).

L'activité lipolytique est réduite et les différences de teneur en acides gras des triglycérides entre le kéfir et le lait utilisé sont minimes (ALM, 1982e). Il en est de même pour l'activité protéolytique qui est plus faible que celle d'autres laits fermentés. A titre d'exemple, ALm (1982f) a constaté que l'azote non protéique du kéfir était passé de $29 \mathrm{mg} / 100 \mathrm{~g}$ dans le lait à $46 \mathrm{mg} / 100 \mathrm{~g}$ dans le kéfir du premier jour et à $52 \mathrm{mg} / 100 \mathrm{~g}$ après 11 jours de conservation. Parmi les acides aminés libres, la thréonine, la proline et la lysine avaient présenté l'augmentation la plus prononcée.

La digestibilité in vitro du kéfir, estimée par la taille des particules du caillé, est importante (Alm, 1982c). Elle est attribuée à la petite taille de ces particules et résulte probablement de l'acidification et de la protéolyse partielle.

Les données concernant le taux de vitamines du kéfir ne sont pas nombreuses. Récemment, Alm (1982b) a révélé que les vitamines $\mathrm{B}_{6}, \mathrm{~B}_{12}$ et la biotine représentaient une réduction de $15 \%$ par rapport au lait cru et que l'acide folique augmentait de $40 \%$ durant le premier jour et de $25 \%$ après une conservation de 11 jours. D'autres travaux, datant de plus de 20 ans, présentent des résultats plus ou moins contradictoires, dûs évidemment à la diversité du lait et de la microflore des kéfirs étudiés, sans négliger le rôle joué par la sensibilité de la méthode d'estimation utilisée. En général, le contenu final en vitamines dépend de l'activité microbienne et résulte de l'équilibre entre leur production et leur consommation par les microorganismes du produit.

On attribue au kéfir un effet favorable ou même préventif dans les cas de plusieurs maladies (anémie, gastrite, diarrhée des nourrissons, maladies intestinales des enfants, problèmes de digestion des personnes âgées), ainsi qu'un effet bénéfique sur l'appareil circulatoire et sur le système nerveux. Préparé à partir du lait écrémé, il pourrait être consommé dans les cas de diabète, d'obésité ou de maladies cardiaques et rénales (Lipatov, 1978). Cependant, à la différence du yoghourt ou d'autres laits fermentés (Anon., 1983b) nous ne disposons que de très peu de données concernant le rôle curatif du kéfir.

La consommation du kéfir n'est pas recommandée durant les phases aiguë et terminale de la pneumonie et de la bronchite de l'enfant et du nourrisson, puisqu'il conduit à un déplacement de l'équilibre acido-alcalin du sang vers l'acidose au moment où la capacité à tamponner de l'organisme est considérablement réduite (Ormisson et Soo, 1976).

Suivant la tendance moderne à étudier l'effet antitumoral des laits fermentés, des chercheurs Japonais (Shiomi et al., 1982 ; Murofushi et al., 1983) ont isolé des grains de kéfir un polysaccharide hydrosoluble, composé de glucose et de galactose dans une proportion de $1: 1,15$. Il présentait une activité inhibitrice in vivo pour les ascites d'Ehrlich (inhibition de 40 à $64 \%$ ) et pour le Sarcome 180 (inhibition de 20 à $90 \%$ ) des souris. 


\section{Conclusion}

Les connaissances actuelles concernant le kéfir mettent en évidence l'intérêt de ce lait fermenté et sa particularité. Celle-ci résulte de la complexité de la microflore utilisée, mais surtout de l'utilisation des grains de kéfir, dont la production in vitro n'a pas été obtenue jusqu'à présent. Par conséquent, l'industrialisation de la production du kéfir devait résoudre le problème d'un coût élevé dû à son levain original. Les innovations mises en œuvre y ont contribué et ont permis l'extension de son marché. Ses propriétés nutritionnelles et éventuellement médicales augmentent sa popularité.

Malgré les nombreux travaux réalisés jusqu'à présent, plusieurs points restent encore à éclaircir : le mécanisme de formation des grains de kéfir, toutes les relations complexes entre les différents groupes microbiens qu'on y rencontre, l'effet éventuel de la boisson sur la santé de l'homme. Les résultats obtenus permettront alors l'optimisation de la production industrielle en conduisant à une meilleure maîtrise de la flore du levain. Parallèlement, ils élimineront les mythes qui sont encore attachés à la consommation du kéfir.

Reçu le 16 février 1987. Accepté pour publication le 18 février 1988.

\section{Remerciements}

Nous tenons à remercier M. J.-P. Accolas de sa lecture critique d'une des nombreuses versions de ce texte, ainsi que M. M. Desmazeaud. Mme Michèle Landon et Mlle Catherine FOUCAUD de leurs suggestions durant la rédaction en français de cet article.

Enfin, nous remercions MM. V. BotTazzl et $F$. BIANCHI de nous avoir aimablement autorisés à reproduire les photos extraites de leur article.

\section{Références bibliographiques}

Alekseev N.G., Gouliaev V.L., 1981. Preparation of cultured milk products for freeze-drying. Molochn. Prom-st., n ${ }^{\circ}$ 4, 18-19 (DSA 1982, $\mathrm{n}^{\circ} 184$ ).

Alm L., 1982a. Effect of fementation on lactose, glucose and galactose content in milk and suitability of fermented milk products for lactose intolerant individuals. J. Dairy Sci., 65, 346-352.

AL.M L., 1982b. Effect of fermentation on B-vitamin content of milk in Sweden. J. Dairy Sci., 65, 353-359.

ALM L., 1982c. Effect of fermentation on curd size and digestibility of milk proteins in vitro of Swedish fermented milk products. $J$. Dairy Sci., 65, 509-514.

Al.m L., 1982d. Effect of fermentation on $\mathrm{L}(+)$ and $\mathrm{D}(-)$ lactic acid in milk. J. Dairy Sci., 65, 515-520.

ALM L., 1982e. Effect of fermentation on milk fat of Swedish fermented milk products. J. Dairy Sci., 65, 521-530.

At.m L, 1982f. Effect of fermentation on proteins of Swedish fermented milk products. J. Dairy Sci., 65, 1696-1704. 
Anonymous a. Dairy Handbook. Published by ALFA LAVAL AB. Dairy and Food Engeneering Division, Lund, Sweden, 181-183.

Anonymous b. [Milk beverages, kefïr]. Poland Polski Komitet Normalizacji Imihr (DSA 1979, $\left.n^{\circ} 183\right)$.

Anonymous, 1969. Norme de composition pour les laits fermentés. Norme Internationale FIL-IDF 47.

Anonymous, 1976. [Cultured milk drinks-a growth market]. German Federal Republic, G. and I. Forschungsgemeinschaft. CMA-Mafo-Briefe (363), 23 pp. From World Agric. Econ. Rural Sociol. Abstr., 19, 237 (DSA 1977, $\mathrm{n}^{\circ} 4656$ ).

Anonymous, 1983a. Introduction générale. FIL-IDF Document 159, 2-4.

Anonymous, 1983b. Influence sur la santé d'une alimentation avec des produits laitiers contenant des cultures de microorganismes. FIL-IDF Document 159, 20-32.

Berzhinskas G., Urbene S., Puidokas I., 1978. Influence of multiple-stage pasteurization of milk on the consistency of kefir. XX Int. Dairy Congr., Brief Comm., Vol. E, 840-841.

Bogdanova E.A., Padaryan I.M., Lavrenova G.S., Inozemtseva V.F., 1982. Use of protein concentrates in the manufacture of dairy products. XX Int. Dairy Congr., Brief Comm., Vol. E, 845.

BONDAREV V.YA., 1981. Effect of changing calcium ions concentration on the development of kefir microflora. Molochn. Prom-st., $\mathrm{n}^{\circ}$ 3, 26-27 (DSA 1982, $\mathrm{n}^{\circ} 495$ ).

BotTAZZı V., B1ANCHI F., 1980. A note on scanning electron microscopy of microorganisms associated with the kefir granule. J. Appl. Bacteriol., 48, 265-268.

Cerna J., Hrabova H., 1977. Biologic enrichment of fermented milk beverages with vitamin $B_{12}$ and folic acid. Milchwissenschaft, 32, 274-277.

Choinowski W., Poznanski S., Bednarski W., Poliwko I., 1979 publ. 1981. Production of cultured beverages from milk and whey concentrated by ultrafiltration. Roc. Inst. Przem + Mlecz., 21, 25-33 (DSA 1982, $n^{\circ} 1478$ ).

Chojnowski W., Poznanski S., Smietana Z., Bednarski W., 1978. Production of fermented products from milk and whey using ultrafiltration. XX Int. Dairy Congr. Brief Comm., Vol. E, 946.

DESEZ M.A., 1964. Synthèse des réglementations connues sur les laits fermentés. FIL-IDF Ann. Bull. - Part. III « Fermented Milks ", 139-140.

Duitschaever C.L., Kemp N., Emmons D., 1987. Pure culture formulation and procedure for the production of kefir. Milchwissenschaft, 42, 80-82.

Eller T., 1983. Stabilizers for flavoured milks. Dtsch. Molk.-Ztg., 104, 4-8.

Gawel J., Gromadka M., 1978. Chemical changes during fermentation and ripening of kefir. XX Int. Dairy Congr., Brief Comm., Vol. E, 839-840.

GLAESER H., 1981. Kefir : cultures, production, chemical composition and nutritive value. Ernähr.Umsch., 28, 156-158 (DSA 1982, $\mathrm{n}^{\circ}$ 6920).

Glaeser H., Beiter M., 1979. Enzymatic determination of ethanol in commercial and laboratorymade kefir. Dtsch. Milchwirtsch., 30, 1865-1868 (DSA 1981, $\mathrm{n}^{\circ} 1056$ ).

Gorner F., PALO V., SEginova M., 1970. Formation of highly volatile compounds in kefir ripening. XVIII Int. Dairy Congr., Vol. 1E, 712.

Gouliaev V.L., Alexeiev N.G., 1982. Intensification of freeze-drying process of cultured milk products. XXI Int. Dairy Congr., Vol. 1, Book 1, 282.

Guigo E.I., Alekseev N.G., Malkov L.S., 1981. Efficient commercial equipment for freeze-drying of foods. Kholod. Tekh., (5), 50-53 (DSA 1982, $\mathrm{n}^{\circ}$ 5224).

HiRota T., 1987. Microbiological studies on kefir grains. Reports of Research Laboratory Snow Brand Milk Products Co, (84), 67-128.

HiRota T., KIKUCHı T., 1976. Studies on kefir grains. II. Some properties of B-galactosidase extracted from kefir grains. Reports of Research Laboratory Snow Brand Milk Products Co, (74), 83-89 (DSA 1981, $\mathrm{n}^{\circ}$ 5423).

Horvath G.Y., 1968. The kefir and its production. Tejipar, 17, 84-88.

IVANOVA L.N., BulatSkaya A.N., Silaev A.E., 1980. Industrial production of kefir for children. Molochn. Prom-st., (3), 15-17 (DSA 1981, $\mathrm{n}^{\circ}$ 106).

Inasawa S., Ueda M., Miyata N., Hirota T., Ahiko K., 1982. Identification and fermentation character of kefir yeast. Agric. Biol. Chem., 46, 2631-2636 (DSA 1983, $\mathrm{n}^{\circ}$ 7219). 
Jacquet J., Thevenot R., 1961. Le lait et le froid, J.B. Baillère, Paris, 216-218.

KARLIN R., 1965. Enrichment of kefir with vitamin $B_{12}$ by the addition of Propionibacterium shermanii. Int. Z. Vitaminforsch., 35, 358-364 (DSA 1966, $\mathrm{n}^{\circ} 1588$ ).

Kemp N., 1984. Kefir, the champagne of cultured dairy products. Cult, Dairy Prod. J., 19, 29-30.

KLyAvinya L.R., 1980. [Department for dairy products for children]. Molochn. Prom-st., (3), 11-12 (DSA 1981, $\left.\mathrm{n}^{\circ} 105\right)$.

Koroleva N.S., 1982. Special products (kefir, koumys, etc.). A lecture. XXI Int. Dairy Congr., Vol. 2, 146-151.

Koroleva N.S., Bavina N.A., 1970. Effects of conditions of kefir fungus cultivation on the microflora and biochemical characteristics of kefir starters. XVIII Int. Dairy Congr., Vol. 1E, 413.

Koroleva N.S., Rozhrova I.V., Bavina N.A., 1978a. Basic factors affecting the microflora and quality of kefir. XX Int. Dairy Congr., Brief. Comm., Vol. E, 843.

Koroleva N.S., Bavina N.A., Rozhkova I.V., 1978b. Changes of microflora of kefir during storage. XX Int. Dairy Congr., Brief Comm., Vol. E, 844 .

Korovkina L.N., Patkul G.M., Maslov A.M., 1976. The effect of $\mathrm{pH}$, cooling method and chemical composition on the viscosity of kefir. Izv. Vyssh. Uchlebnykh Zaved., Pishch. Tekhnol., (5), 30-32 (DSA 1977, n० 5284).

Kosikowskı F.V., 1977. Cheese and fermented milk foods, 2nd edition. Edwards Brothers, Inc. Ann Arbor, Michigan, 40-42.

Kramkowska A., Kornacki K., Bauman B., Fesnak D,, 1982. Method of production of a single-use kefir culture. XXI Int. Dairy Congr., Brief Comm., Vol. 1, Book 2, 304-305.

Lang F., LANG A., 1973. Cultured milk products. Food Manuf., 48, 23-28, 54.

La Riviere J.W.M., Koolman P., Schmidt K., 1967. Kefiran, a novel polysaccharide produced in the kefir grain by Lactobacillus brevis. Arch. Microbiol., 59, 269-278.

Lipatov N.N., 1978. Fermented milks other than yoghurt. A lecture. XX Int. Dairy Congr., Sessions Scientifiques et Techniques, ST 43, $15 \mathrm{pp}$.

Marshall V.M., Cole W.M., 1984. Studies on kefir. IDF Bull, 179, XIII.

Marshall V.M., COLE W.M., 1985. Methods for making kefir and fermented milks based on kefir. J. Dairy Res., 52, 451-456.

Merilainen V.T., 1984. Microorganisms in fermented milks : other microorganisms. IDF Bull., 179, 89-93.

MOLSKA I., KOCON J., ZMARLICKI S., 1980. Electron microscopic studies on structure and microflora of kefir grains. Acta Aliment. Pol., 6, 145-154 (DSA 1981, n 7063).

Moulin G., Arthaud J.F., Ratomahenina R., Galzy P., 1977. Notes on kefir production. Ind. Alim. Agric., 94, 495-497.

Murofushi M., Shiomi M., Aibara K., 1983. Effect of orally administered polysaccharide from kefir grain on delayed-type hypersensitivity and tumor growth in mice. Jap. J. Med. Sci. Biol., 36, 49-53 (DSA 1985, n 5609).

Ohara N., Kozaki M., Kitahara K., 1977. Microbiological studies on kefir I. Isolation and identification of yeasts from kefir. J. Agric. Sci., 21, 92-103 (DSA 1978, n 6019).

Ormisson A.A., Soo T.R., 1976. Effect of soured milk and kefir on indices of acide-base balance of arterial blood in healthy infants and in infants with acute pneumonia and bronchitis. Pediatr. (Moscow), $\mathrm{n}^{\circ}$ 10, 37-38 (DSA 1977, $\mathrm{n}^{\circ}$ 2757).

Ottogalli G., Galli A., Resmini P., Volonterio G., 1973. Composizione microbiologica, chimica ed ultrastruttura dei granuli di kefir. Ann. Microbiol., 23, 109-121.

Petricic A., Grüner M., Jacopovic L., 1977. Effect of processing conditions on ethanol content and acidity of kefir. Mljekarstvo, 27, 181-185 (DSA 1978, $\mathrm{n}^{\circ} 2878$ ).

Pettersson H.E., 1984. Freeze-dried concentrated starter for kefir. IDF Bull. 179, XVI-XVII.

Pettersson H.E., Christiansson A., Ekelund K., 1985. Making kefir without grains. Scand. J. Dairy Technol. Know-How, $\mathrm{n}^{\circ} 2,58-60$.

Pidoux M., 1984. Les kéfirs - kéfirs lactés et kéfirs sucrés. Tech. Lait, n² 22, 29-33.

Piechocka M., Holmen T.B., Abrahamsen R.K., 1977. Heat treatment, homogenization and incubation of milk for production of kefir of various fat levels. Nordeuropaeisk Mejeri-Tidsskr., 43, 334-343. 
Rogosa M., 1974. Genus I. Lactobacillus. In Bergey's Manual of Determinative Bacteriology. R.E. BUCHANAN \& N.E. GIBBONS (eds), Williams \& Wilkins, Baltimore, 577-583.

Roczniakova B., Kornacka D., Bielecka M., 1974. Stimulatory effect of the microflora of kefir grains on the biochemical activity of propionic acid bacteria. XIX Int. Dairy Congr., Vol. 1E, $387-388$.

Rosı J., 1978a. I microorganismi del kefir: I lieviti. Sci. Tec. Latt.-Casearia, 29, 59-67.

Rosı J., 1978b. I microorganismi del kefir: Gli acetobatteri. Sci. Tec. Latt.-Casearia, 29, 221-227.

Rosı J., Rossı J., 1978. I microorganismi del kefir : I fermenti lattici. Sci. Tec. Latt.-Casearia, 29, 291-305.

Rosı J., Rossı J., 1980. Le proteine del siero recuperate per ultrafiltrazione, nell'ottenimento del kefir e come mezzo per valutare la riproduzione dei granuli. Sci. Tec. Latt. - Casearia, 31, 241-251.

Rosı J., Rossı J., 1981. Condizioni per la produzione di polysaccaride in alcuni Lactobacilli, in relazione alla formazione del granulo di kefir. Atti del XLI Convegna della sezione Tosco-UmbraEmiliana-Marchigiana-abruzzese (TUEMA) della Società Italiana di Microbiologia, Siena, 29-30 Maggio 1981.

Rossi J., Costamagna L., INGI M., 1978. Boissons fermentées de différents types microbiens (L. acidophilus, L. bifidus, L. bulgaricus et Str. thermophilus) produites avec du lait à faible contenu glucidique (Lait ultrafiltré, lait délactosé par voie microbienne, lait de soja). Lait, 58, 155-172.

Rossı J., Clementı F., 1983. Bevande fermentate prodotte con substrati non convenzionali. Sci. Tec. Latt. - Casearia, 34, 325-335.

Shiomi M., Sasaki K., Murofushi M., Aibara K., 1982. Antitumor activity in mice orally administered polysaccharide from kefir grain. Jap. J. Med. Sci. Biol., 35, 75-80 (DSA 1985, n ${ }^{\circ}$ 5608).

Siegenthaler M.E., Ritter T., 1964. L'emballage des laits fermentés. FIL-IDF Ann. Bull. - Part III "Fermented Milks », 113-114.

SiEgLER K.U., 1982. Cultured milk beverages - a market segment with atractive growth prospects. Dtsch. Milchwirtsch., 33, 265-267.

Szakaly S., Obert G., Al-Khafajl K.M., 1982, Effect of homogenization on the keeping quality of sour milk products. XXI Int. Dairy Congr., Vol. 1, Book 1, 291.

VAYSSIER Y., 1978. Le kéfir : Étude et mise au point d'un levain pour la préparation d'une boisson. Rev. Lait, Fr., (362), 131-134.

Wiesner H.-U., Stahlhut-Klipp H., Benner J., 1975. Occurrence of D(-) lactate in fermented milk, yoghurt and kefir. Arch. Lebensmittelhyg., 25, 49-52 (DSA 1975, $\mathrm{n}^{\circ}$ 7235). 